\title{
Andreas Vesalius (1514-1564). Fundador de la Anatomía Humana Moderna
}

\author{
Andreas Vesalius (1514-1564). Founder of the Modern Human Anatomy
}

\author{
Rafael Romero Reveron
}

ROMERO, R. R. Andreas Vesalius (1514-1564). Fundador de la Anatomía Humana moderna. Int. J. Morphol., 25(4):847-850, 2007.

RESUMEN: Andreas Vesalius, (Andries van Wesel), nació en Bruselas, Flandes, actual Bélgica, el 31 de diciembre de 1514. Estudió Medicina en las Universidades de Lovaina, Paris y Papua, donde se doctoró, graduándose magna cum laude, el 5 de diciembre de 1537, a los 23 años de edad; al día siguiente fue nombrado explicator chirugiae, el equivalente a una cátedra de Cirugía y Anatomía, en la misma Universidad. Durante sus demostraciones prácticas él mismo hacía sus disecciones anatómicas, en lugar de confiárselas a un cirujano, rompiendo con la tradición. Vesalio estudió y develó la Anatomía Humana más que todos sus predecesores al crear, en 1543, De humani corporis fabrica (Sobre la estructura del cuerpo humano), el cual puede considerarse como el primer tratado moderno de Anatomía, tanto por su claridad como por el rigor expositivo de sus contenidos y ha sido uno de los libros más influyentes sobre anatomía humana. Vesalio es considerado el fundador de la anatomía moderna por sus aportes significativos realizados en la enseñanza y aprendizaje de la anatomía humana. Posteriormente, siguiendo la tradición familiar, Vesalio fue requerido por el rey español Carlos V para que formara parte de su servicio médico; por tanto, marchó a Bruselas. Allí se casó, ejerció la medicina y continuó escribiendo. Luego se trasladó a Madrid donde, en 1556, obtuvo el título de Conde Palatino del Rey Felipe II, quien logró que le fuera conmutada la pena de muerte por haber iniciado una observación anatómica interna en un hombre vivo, por una peregrinación a Jerusalén, pero durante el viaje de regreso su barco naufragó cerca de la isla griega de Zante, a donde le arrastraron las aguas y en la que murió el 15 de octubre de 1564.

PALABRAS CLAVE: Andreas Vesalius; Anatomía humana; De humani corporis fabrica; Disecciones anatómicas; Enseñanza y aprendizaje.

Andreas Vesalius (Andries van Wesel, Andrés Vesalio) estudió y develó la anatomía humana más que todos sus predecesores al crear, en1543, De humani corporis fabrica (Sobre la estructura del cuerpo humano) que, como todos sus trabajos, puede calificarse de "renacentista" por su forma de ver y describir el cuerpo humano, una edificación estática, una "fábrica" o edificio. Frente a la confusión entre "forma" y "función" de Galeno y toda la morfología tradicional, Vesalio distingue cuidadosamente ambos aspectos de la realidad, dando una visión estática del organismo humano.

Su obra puede considerarse como el primer tratado moderno de anatomía humana, tanto por su claridad como por el rigor expositivo de sus contenidos y es uno de los libros más influyentes sobre este tema, por lo que me interesé en investigar y recopilar la información consultada, para realizar esta síntesis biográfica de algunos aspectos de interés de su vida y obra, como parte de una línea de investigación en desarrollo sobre personas que han realizado aportes significativos en la enseñanza y aprendizaje de la anatomía humana, como un conocimiento complementario.

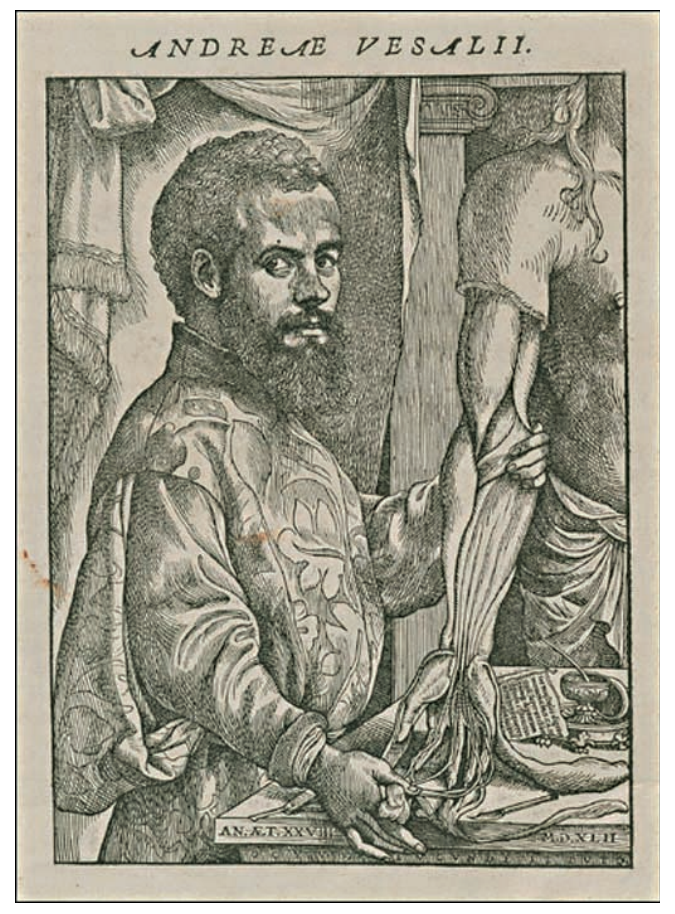


Andrés Vesalio, la forma latinizada del nombre Andries van Wesel, nació en Bruselas, Flandes, que por entonces, formaba parte del Sacro Imperio Romano Germánico, actual Bélgica, 31 de diciembre de 1514, anatomista flamenco, procedía de una estirpe renana consagrada en sus últimas generaciones al servicio médico de los emperadores de Alemania. Recibió su primera educación en Bruselas y Lovaina donde, entre otras materias, aprendió latín y griego. Admiró desde pequeño la obra biológica de Alberto Magno y tuvo cierta tendencia a la disección de animales.

Su padre Andries, era hijo ilegítimo del médico real del emperador Maximiliano I, Everard Van Wesel. Andries sirvió también a Maximiliano como boticario, y luego a su nieto Carlos V, como valet de chambre. Animó a su hijo a seguir la tradición familiar y lo inscribió en la Escuela de los Hermanos de la Vida Común, en Bruselas, donde Vesalio aprendió griego, árabe, hebreo y latín. En 1528, Vesalio ingresó a la Universidad de Lovaina (Pedagogium Castrensis) como estudiante de artes pero, cuando su padre fue nombrado valet de chambre, en 1532, prefirió seguir estudios de medicina en la Universidad de París, ciudad a la que se trasladó en 1533. Allí estudió las teorías de Galeno, bajo la dirección de Jacobus Sylvius y de Jean Ferne. Fue en esa época cuando empezó a interesarse por la anatomía y para mejorar sus conocimientos sobre osteología se proveía de huesos en el Cementerio de los Inocentes.

Se vio obligado a abandonar París en 1536, a causa de la guerra entre Francia y el Sacro Imperio Romano Germánico, y regresó a Lovaina. Allí completó sus estudios bajo la dirección de Johannes Winter von Andernach, y se graduó al año siguiente. Permaneció en Lovaina poco tiempo, a causa de una disputa con su maestro. Se instaló transitoriamente en Venecia y en 1536 se trasladó a la Universidad de Padua, donde se doctoró, en 1537. Se graduó magna cum laude el 5 de diciembre de 1537, a los 23 años de edad. Al día siguiente de su graduación fue nombrado explicator chirugiae, el equivalente catedrático de Cirugía y Anatomía, en la misma Universidad y empezó a dar conferencias a los estudiantes. Impartió también lecciones en las universidades de Bolonia y de Pisa. Su método de enseñanza era revolucionario: tradicionalmente el profesor enseñaba leyendo los textos clásicos (principalmente la obra de Galeno) y su exposición era seguida de la disección de un animal, realizada por un barberocirujano bajo la dirección del profesor; Vesalio, en cambio, convirtió la disección en la parte más importante de la clase, llevándola a cabo por sí mismo, rodeado por sus alumnos.

Para Vesalio, la observación directa era la única fuente fiable, lo que suponía una importante ruptura con la práctica medieval, basada fundamentalmente en los textos. Para sus demostraciones prácticas de anatomía, Vesalio rompió con la tradición y él mismo hacía sus disecciones, en lugar de confiárselas a un cirujano. Recopiló sus dibujos de anatomía, para uso de sus estudiantes, en tablas anatómicas ilustradas. Cuando descubrió que algunos de ellos estaban siendo copiados, los publicó en 1538 con el título de Tabulae Anatomicae Sex (Venecia, 1538). Tres láminas osteológicas fueron realizadas por Kalkar, a instancias suyas; tres relativas a vísceras (hígado, porta y genitales; hígado y cava; corazón y aorta) las realizó él mismo. En 1538, publicó una obra sobre la flebotomía o sangría, que era en la época un tratamiento que se aplicaba a casi cualquier enfermedad, existiendo un debate acerca de cuál era el lugar más adecuado para la extracción de sangre. El procedimiento defendido por Galeno, consistía en extraer la sangre de un punto cercano al de la localización de la enfermedad. Sin embargo, la práctica musulmana y medieval prescribía extraer menor cantidad de sangre de un punto más distante. La obra de Vesalio apoyaba la opinión de Galeno, basando sus argumentos en diagramas anatómicos.

En 1539, un juez de Padua, interesado por los trabajos de Vesalio, hizo que se le facilitasen los cadáveres de los criminales ejecutados, para la disección. Esto le permitió mejorar sus diagramas anatómicos. En 1539, con el fin de aportar claridad a una polémica sobre la sangría en las afecciones neumónicas monolaterales, el médico de Carlos V, Nicolás Florena, encargó a Vesalio un exploración disectiva del sistema venoso endotorácico. Descubrió así, la vena ácigos mayor y su desembocadura en la vena cava superior (si seguimos la idea galénica de la circulación de la sangre, sería el origen y no el final). Publicó los resultados ese mismo año (Epistola docens venam axillarem dextri cubiti in dolre laterali secandam) y también aceptó el encargo de la Giunta, una afamada casa editorial veneciana, para revisar la edición latina de varios escritos anatómicos de Galeno, concordando con una de las características del Renacimiento: revisar y corregir. Concluyó el trabajo apenas un año después. En 1539, publicó una versión actualizada del vademécum anatómico de Galeno, Institutiones Anatomicae.

Todas estas actividades fueron muy apreciadas por el claustro de profesores y por los estudiantes. En el documento oficial que prorroga el nombramiento de Vesalio, se dice claramente que «había suscitado gran admiración entre todos los estudiantes». El estrecho contacto con Galeno a través de sus obras, le llevó a darse cuenta de que dejaba traslucir en sus escritos su experiencia disectiva con monos.

$\mathrm{Al}$ comprobar personalmente en las disecciones tantos errores, en 1541, mientras estaba en Bolonia, Vesalio descubrió que las investigaciones de Galeno estaban basadas en la disección de animales, y no de seres humanos. Como la disección humana había estado prohibida en la antigua Roma, 
Galeno había disecado en su lugar, monos de Berbería, creyendo que serían anatómicamente similares al ser humano. Vesalio abandonó entonces, al indiscutible Galeno de la enseñanza de la anatomía. El hecho causó cierto revuelo en el claustro de Papua, pero Vesalio se comprometió a escribir un nuevo tratado de anatomía.

Vesalio, apoyándose en sus propias observaciones, publicó una corrección de las Opera omnia de Galeno, y comenzó a escribir su propio texto de anatomía. En 1543, ya estaba redactado su conocido De humani corporis fabrica libri septem (Sobre la estructura del cuerpo humano). Dicha obra puede considerarse como el primer tratado moderno de anatomía, tanto por su claridad como por el rigor expositivo de sus contenidos y uno de los libros más influyentes sobre anatomía humana. Basó sus estudios anatómicos en la observación directa, rechazando algunos errores anatómicos presentes en la obra de Galeno, por lo que es considerado el fundador de la anatomía moderna. Deben resaltarse especialmente los grabados de la Fabrica vesaliana. Son magníficos por su calidad, su exactitud anatómica, y la elegante y un poco teatral belleza que supo darles su autor, los de Jan Stefan van Kalkar, así como por su gran número, más de trescientos dibujos.

Los siete libros o partes de que se compone la Fabrica se consagran, los dos primeros, a la exposición de los que pueden llamarse sistemas constructivos del organismo (huesos y cartílagos, ligamentos y músculos); los libros tercero y cuarto tratan de los sistemas conjuntivos: venas, arterias y nervios; los tres últimos se ocupan de la descripción morfológica de los órganos de la nutrición y generación y de los instrumentos de la facultas vitalis, cerebro y sentidos. En la descripción de los órganos internos, la exposición de Vesalio es nueva, renovadora en la explicación anatómica. Además de saber más y mejor la anatomía, Vesalio enseña a saberla de otro modo, el modo correspondiente a la intuición renacentista de la realidad visible, en general, y del cuerpo humano, en particular.

También en 1543 , terminó el Epitome, una especie de Fabrica compendiada para uso de los estudiantes. El texto, al que se unieron trescientas planchas grabadas en madera por Kalkar. La Fabrica iba dedicada al emperador Carlos V y el Epitome al que después sería Felipe II. Vesalio tenía entonces 29 años. La obra originó una reacción airada de algunos galenistas. Uno de los que le atacó ferozmente fue su maestro parisino Jacobo Silvio, quien le propinó calificativos como desvergonzado, impío, calumniador e ignorante.

Vesalio, impertérrito, continuó provocando controversias, esta vez no demostrando los errores de Galeno, sino de Mondino de Liuzzi, e incluso de Aristóteles: los tres habían hecho suposiciones acerca de las funciones y estructura del corazón que eran claramente erróneas. Por ejemplo, Vesalio descubrió que el corazón tenía cuatro cavidades, que el hígado tenía dos lóbulos y que los vasos sanguíneos comenzaban en el corazón, y no en el hígado.

Andrés Vesalio regresó a Padua pero, siguiendo la tradición familiar fue requerido por Carlos $\mathrm{V}$ para que formara parte de su servicio médico; por tanto, marchó a Bruselas. Allí se casó, ejerció la medicina y escribió. Publicó un opúsculo sobre el uso de la raíz de China o zarzaparrilla, donde aprovechó para defenderse de quienes le atacaban por abandonar a Galeno. Mientras preparaba la segunda edición de la Fabrica (1551), también acompañaba al emperador en sus viajes; siendo su función la de médico "internista" y no de cirujano.

Tras abdicar Carlos V, en 1556, pasó al servicio de Felipe II, traslandándose a Madrid en 1559. Su estancia no fue demasiado grata por un desgraciado acontecimiento con el Infante, las relaciones con el resto de los médicos de la casa real y, quizás, por la ausencia de cadáveres para disección. Tuvo en España, sin embargo, ardientes defensores como Pedro Jimeno y Luis Collado, profesores en la Universidad de Valencia.

En 1556, obtuvo el título de Conde Palatino, y tras la abdicación del Emperador continuó prestando sus servicios en la corte de Felipe II, por lo que residió en España la mayor parte del tiempo. Un importante episodio de su labor profesional, como cirujano, es su intervención en el tratamiento de la herida sufrida por el príncipe Carlos.

En 1561, encontrándose en Madrid, fue juzgado por un tribunal de la Inquisición y condenado a muerte por haber iniciado una observación anatómica interna en un hombre vivo. Felipe II logró que le fuera conmutada la pena por una peregrinación a Jerusalén, pero durante el viaje de regreso su barco naufragó cerca de la isla griega de Zante, a donde le arrastraron las aguas y en la que murió, el 15 de octubre de 1564.

Cinco puntos se destacan como los más significativos en el gran aporte que Andrea Vesalio hizo a la enseñanza y aprendizaje de la anatomía humana.

$1^{\circ}$ Sustituye la anatomía libresca de Galeno por otra más fiel a la realidad y basada en su propia experiencia de disector. A Vesalio puede calificársele de disector, profesor, demostrador y dibujante. $2^{\circ}$ Corrige casi todos los errores descriptivos de Galeno. Las descripciones de Vesalio se refieren a la disección del cuerpo humano, no al cuerpo del mono o del perro. $3^{\circ}$ Añade muchos descubrimientos nuevos. $4^{\circ}$ Describe con claridad las partes anatómicas del cuerpo humano; $5^{\circ}$ Utiliza con esplendidez, belleza y eficacia inéditas, la ilustración anatómica. 
ROMERO, R. R. Andreas Vesalius (1514-1564). Founder of the modern Human Anatomy. Int. J. Morphol., 25(4):847-850, 2007.

Andreas Vesalius,(Andries van Wesel), was born in Brussels, Flandes, present Belgium, 31 of December of 1514.He studied Medicine in the Universities of Lovaina, Paris and Papua, , graduating magna cum in December 5, 1537 , to the 23 years of old; where he became explicator chirugiae equivalent to a chair of Surgery and the Anatomy. He teached in the university of Padua. During his practical demonstrations Vesalius broke with the tradition and does himself its anatomy dissections, instead of trusting to a surgeon; Vesalio studied more and reveal the Human Anatomy that all its predecessors when creating in 1543 De humani corporis fabrica (On the structure of the human body), this can be considered like the first modern treaty of anatomy, as much by his clarity as by the exposition of his contents and one of the most influential books on human anatomy, reason why Vesalius is considered the founder of the modern anatomy, by his significant contributions made in the education and learning of the Human Anatomy. Vesalius following the familiar tradition was required by Spanish King Carlos V and by his son Felipe II and marched to Brussels. where He married, and continue his practice of medicine, and his writing,He came to Spain,later. With King Felipe II's influence Vesalius exchanged the capital punishment to have initiated an internal anatomical observation in an alive man by a peregrination to Jerusalem, but during the return trip his boat was shipwrecked near the Greek island of Zante, and Vesalius died in October, $15,1564$.

KEY WORDS: Andreas Vesalius; Human anatomy; De humani corporis fabrica; Anatomy dissections; Education and learning.

\section{REFERENCIAS BIBLIOGRÁFICAS}

AndreVesalius. http://www.artehistoria.com/historia/personajes/6259.htm

A ndrea Ves a lio.( $1514-1564)$. h t t p:// www.historiadelamedicina.org/vesalio.html

AndresVesalio. http://www.hipocrates.tripod.com/historia/ versalio.htm

Biografia de AndreaVesalio. http://it.wikipedia.org/wiki/ Andrea_Vesalio

La Figura deVesalio.http://escuela.med.puc.cl/publ/ HistoriaMedicina/Figuras Vesalio.html

La Figura de Vesalius.http://www.upf.edu/materials/fhuma/ revolucio/treballs/rua/rua.htm\#vesalio

La Medicina del Renacimiento. El Empuje de la Anatomía http://www.ujaen.es/investiga/cts $380 /$ historia/ la_medicina_del_renacimiento.htm

Vasconcellos, Henrique Ayres de y Barros de Vasconcellos, Pedro Henrique. Andrés Vesalio: La articulación témporomandibular. Int. J. Morphol., 24(1):105-9, 2006.

Vesalius Gallery. http://archive.nlm.nih.gov/proj/ttp/ vesaliusgallery.htm
Dirección para correspondencia:

Dr. Rafael Romero Reveron

Cátedra de Anatomía Normal

Facultad de Medicina

Escuela de Medicina José María Vargas

Universidad Central de Venezuela

VENEZUELA

Email:rafa1636@yahoo.es

Recibido : 24-05-2007

Aceptado: 18-10-2007 